\title{
Effective parameters of metal-dielectric composites. Influence of eddy currents due to density fluctuations.
}

Alexey P. Vinogradov ${ }^{1}$, Nawaz Borokur ${ }^{2}$ and Saïd Zouhdi ${ }^{2}$

Corresponding author: Prof. Saïd ZOUHDI

Address: Laboratoire de Génie Electrique de Paris LGEP-Supélec

Plateau de Moulon 91192, GIF-SUR-YVETTE CEDEX

France

E-mail: $\quad \underline{\text { sz@ccr.jussieu.fr }}$

Tel.: $\quad$ +33169851660 


\title{
Effective parameters of metal-dielectric composites: influence of eddy currents due to density fluctuations.
}

\author{
Alexey Vinogradov ${ }^{1}$, Nawaz Borokur ${ }^{2}$ and Saïd Zouhdi ${ }^{2}$ \\ ${ }^{1}$ Institute of Theoretical and Applied Electromagnetism (ITAE), \\ Russian Academy of Sciences, \\ Izhorskaya 13/19, Moscow, 127412, Russia. \\ E-mail: A-Vinogr@yandex.ru \\ ${ }^{2}$ Laboratoire de Génie Electrique de Paris \\ Plateau de Moulon, 91192 Gif-Sur-Yvette Cedex, France. \\ E-mail : $\underline{\text { sz@eccr.jussieu.fr }}$
}

\begin{abstract}
It is shown that the space fluctuations of concentration of conducting inclusions might be responsible for the well-known disagreement between theory and experiment at determining microwave losses in metal-dielectric mixture: the theories (percolation theory, effective medium theory etc.) predict much lower losses than those measured in experiment. It is demonstrated that if the effective skin depth in the regions occupied by the fluctuation is comparable to the mean diameter of these regions we can expect additional losses.
\end{abstract}

PACS. 72.80.Tm Composite materials - 73.43.Cd Theory and modelling

\section{Introduction}

Low loss high-dielectric constant materials are of great interest for radio-physical applications. These materials may be used in designing millimeter-wave filters, for cellular phone antennas, dielectric spacers and so on. In this field, metal-dielectric mixtures manufactured of low loss plastic are of special significance due to the simplicity of their manufacturing and processing.

According to the percolation theory a metal-dielectric mixture can exhibit high values for the real part of the permittivity [1]. This phenomenon appears at low frequencies near the percolation threshold $p_{c}$ [2]. In addition, just below the percolation threshold, the static conductivity is still equal to zero and it is reasonable to expect small losses at least at low frequencies. Below the percolation threshold the losses appear due to Maxwell displacement currents that connect those conducting clusters, which tend to form an infinite percolation cluster. It is the current flowing through the chain of big clusters and narrow dielectric gaps that determines the dissipation in the whole system [1,2]. The increase of frequency causes the growth of Maxwell displacement currents. As a consequence more and more clusters join in the current transport which results in additional losses. At certain frequencies the modulus of the complex conductivity $i \varepsilon_{d} \varepsilon_{0} \omega$ of the dielectric matrix becomes comparable to the effective conductivity of conducting clusters. It means that all the clusters are involved in electrical transport and we cannot expect an additional increase of losses. The percolation theory predicts a remarkable frequency dispersion of the real part of permittivity and the maximum of losses to be at frequency $\omega$ where $\varepsilon_{d} \varepsilon_{0} \omega \sim \sigma_{m}$, (in CGS system $\omega$ is about the conductivity $\sigma_{m}$ of the metal 
component). For example, for copper inclusions embedded in dielectric matrix with $\varepsilon_{d} \sim 3$ we get $\omega \approx \sigma /\left(\varepsilon_{d} \varepsilon_{0}\right)=\frac{1}{\left(\rho_{\mathrm{Cu}}=1.6 * 10^{-8} \Omega \mathrm{m}\right)} \frac{1}{3 * 8.854 * 10^{-12} \mathrm{~F} / \mathrm{m}}=\left[\frac{1}{\Omega} \frac{1}{\mathrm{~m}} \frac{\mathrm{m}}{\mathrm{F}}\right]=3 * 10^{18}[\mathrm{~Hz}]$ (note that $\left.\mathrm{F}=\mathrm{m}^{-2} \cdot \mathrm{kg}^{-1} \cdot \mathrm{s}^{4} \cdot \mathrm{A}^{2}, \quad \Omega=\mathrm{m}^{2} \cdot \mathrm{kg} \cdot \mathrm{s}^{-3} \cdot \mathrm{A}^{-2}\right)$. The losses rapidly fall down as the frequency decreases [1, 3] and in the micro wave range $\left(10^{6}-10^{10} \mathrm{~Hz}\right)$ the percolation theory predicts a low level of dissipation, acceptable for applications.

Unfortunately, the dissipation observed in practice significantly exceeds the prediction of the percolation theory: on one hand, the maximum of losses lies at much lower frequencies than those predicted by the theory, on the other hand, this maximum is broader than the expected one. This effect is usually related to the contact resistance between inclusions without any discussion of the origin of this resistance. In this paper we suggest a mechanism of energy dissipation that does not mean an introduction of any hypothetic contact resistance. This mechanism takes place at frequencies significantly lower than that defined by the metal conductivity. The mechanism is connected with the skin effect.

\section{Penetration depth and density effect on permittivity}

It is well known that, at high frequencies, the current penetrates a metal inclusion to the thickness $\delta=c / \sqrt{2 \pi \sigma \omega}$ (skin depth). As a consequence, the effective conductivity of an inclusion of radius $a_{i n c l}$ decreases by a factor of $\delta / a_{i n c l}$. Indeed, if we consider the wave length in matrix to be much greater than all other scales (inclusion size, correlation length etc.) then in the matrix the disturbance of the applied field $\vec{E}_{0}$ can be searched in the dipole form:

$$
\varphi=-\alpha V \vec{E}_{0} \cdot \nabla\left(\frac{1}{r}\right), \quad \vec{E}_{e x t}=\frac{V \alpha_{E}}{r^{3}}\left[3 \vec{n}\left(\vec{n} \cdot \vec{E}_{0}\right)-\vec{E}_{0}\right]+\vec{E}_{0},
$$

where $\alpha_{E}$ is the polarization of the sphere of volume $V$. The fields inside the inclusion are governed by the Maxwell equations

$$
\begin{gathered}
\Delta \vec{E}_{i}+k^{2} \vec{E}_{i}=0, \\
\mathcal{E}_{i} \operatorname{div} \vec{E}_{i}=0 .
\end{gathered}
$$

Eq. (1b) means that we can introduce a vector $\vec{A}\left(\vec{E}_{i}=\operatorname{curl} \vec{A}\right)$. Due to spherical symmetry of the inclusion $\vec{A}$ has the form of $\beta_{E} \operatorname{curl}\left(f(r) \vec{E}_{0}\right)$, where $f(r)=\sin k r / r^{1}$ is a solution to the wave equation (see [4]). Thus, we arrive at the following expression for the internal field:

$$
\vec{E}_{i}=\beta_{E}\left(f^{\prime} / r+k^{2} f\right) \vec{E}_{0}-\beta_{E}\left(3 f^{\prime} / r+k^{2} f\right) \vec{n}\left(\vec{E}_{0} \cdot \vec{n}\right) .
$$

Taking into account that at the surface of the inclusion the tangential components of the electrical and magnetic fields as well as normal components of the electrical and magnetic induction are continuous we obtain equations to determine $\alpha_{E}, \beta_{E}$ :

\footnotetext{
${ }^{1}$ In this approximation the electrical and magnetic problems are solved separately. We can write the identical equation to find $\left(\alpha_{H}, \beta_{H}\right)$. Certainly the found fields $\vec{E}_{i}$ and $\vec{H}_{i}$ induce secondary fields $\vec{H}_{1}=(i \omega / c \mu) \operatorname{curl} \vec{E}_{i}$ and $\vec{E}_{1}=-(i \omega / \varepsilon c) \operatorname{curl} \vec{H}_{i}$ respectively. It has been shown [3,5] that these fields should be neglected in the homogenization problem. Thus, we can confine to the fields $\vec{E}$ and $\vec{H}$.
} 


$$
\begin{gathered}
\varepsilon_{\text {ext }}\left(1+2 \frac{\alpha_{E}}{a_{i n c}^{3}}\right)=\varepsilon_{\text {int }} \beta_{E}\left(-2 \frac{f^{\prime}}{a_{i n c l}}\right), \\
1-\frac{\alpha_{E}}{a_{i n c l}^{3}}=\beta_{E}\left(\frac{f^{\prime}}{a_{i n c l}}+k_{i}^{2} f\right) .
\end{gathered}
$$

Solving these equations we arrive at the renormalized expression for dipole moment:

$$
\frac{\alpha_{E}}{a_{i n c l}^{3}}=\frac{\varepsilon_{\text {int }} F\left(k_{\text {int }} a_{i n c l}\right)-\varepsilon_{\text {ext }}}{\varepsilon_{\text {int }} F\left(k_{\text {int }} a_{i n c l}\right)+2 \varepsilon_{\text {ext }}},
$$

where $F=2 F_{1} /\left(1-F_{1}\right), F_{1}(x)=\frac{1}{x^{2}}-i \frac{e^{i x}+e^{-i x}}{x\left(e^{i x}-e^{-i x}\right)}$.

This fact may be taken into account by the Garnett approximation [6] while calculating the dipole moments of metallic inclusions or by the effective medium theory (EMT) [3, 7] ] while calculating the dipole moments of the metallic and dielectric inclusions (see also [8]). Below we employ EMT approach. The index " $m$ " stands for a metallic inclusion with permittivity $\varepsilon_{m}=$ $i 4 \pi \sigma / \omega, \sigma$ is the conductivity and $\omega$ is the radian frequency. The index " $d$ " stands for a dielectric inclusion with permittivity $\varepsilon_{d,} k_{\mathrm{m}}=(1+i) / \delta_{\mathrm{m}}, \delta_{m}=c / \sqrt{2 \pi \sigma_{m} \omega}$.

The usual EMT assumes that the mean dipole moment of inclusions embedded in a uniform medium with permittivity $\varepsilon_{\text {eff }}$ is equal to zero:

$$
p \frac{\varepsilon_{m}-\varepsilon_{e f f}}{\varepsilon_{m}+2 \varepsilon_{e f f}}+(1-p) \frac{\varepsilon_{d}-\varepsilon_{e f f}}{\varepsilon_{d}+2 \varepsilon_{e f f}}=0,
$$

where $p$ is the volume fraction of metallic inclusions. This equality serves to find the value of $\varepsilon_{\text {eff }}$. The skin effect results in a modification of the dipole moment expression according to Eq. (2). Substituting the modified expressions of the dipole moments into Eq.(3) yields to the equation of the modified effective medium theory (MEMT) (see $[3,7]$ for details):

$$
p \frac{\varepsilon_{m} F\left(k_{m} a_{m}\right)-\varepsilon_{e f f}}{\varepsilon_{m} F\left(k_{m} a_{m}\right)+2 \varepsilon_{e f f}}+(1-p) \frac{\varepsilon_{d} F\left(k_{d} a_{d}\right)-\varepsilon_{e f f}}{\varepsilon_{d} F\left(k_{d} a_{d}\right)+2 \varepsilon_{e f f}}=0 .
$$

Comparing (4) to the usual EMT formula (3) one can see that the skin effect leads to the renormalization of the inclusion's permittivity: instead of $\varepsilon_{m}$ one obtains $\varepsilon_{m}^{(\bmod )}=\varepsilon_{m} F\left(k_{m} a_{m}\right)$. A proper branch of the solution to the Eq. (4) is produced by the following expression [9]:

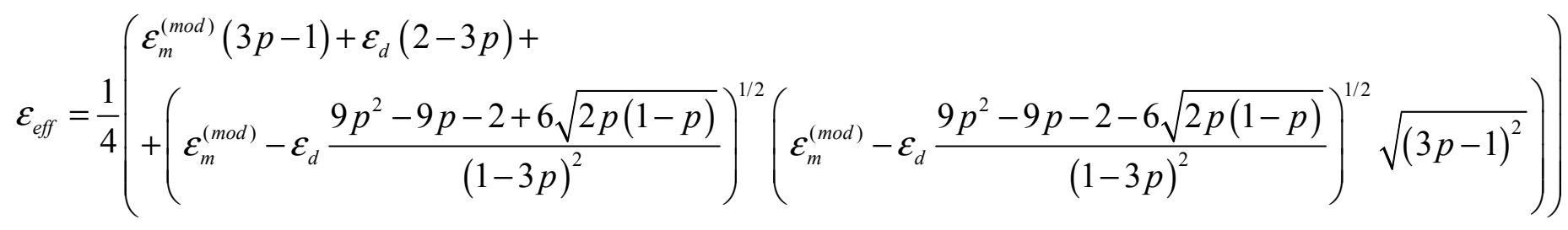

where the single-valued branch of $\sqrt{z}$ is defined as $\sqrt{|z|} \exp (i \arg (z) / 2)$ with a cut along negative real axis. We have taken into account that in our case $k_{d} a_{d}=\varepsilon_{d} a_{d} c / \omega<<1$ and $\mathrm{F}\left(k_{d} a_{d}\right) \approx 1$. 


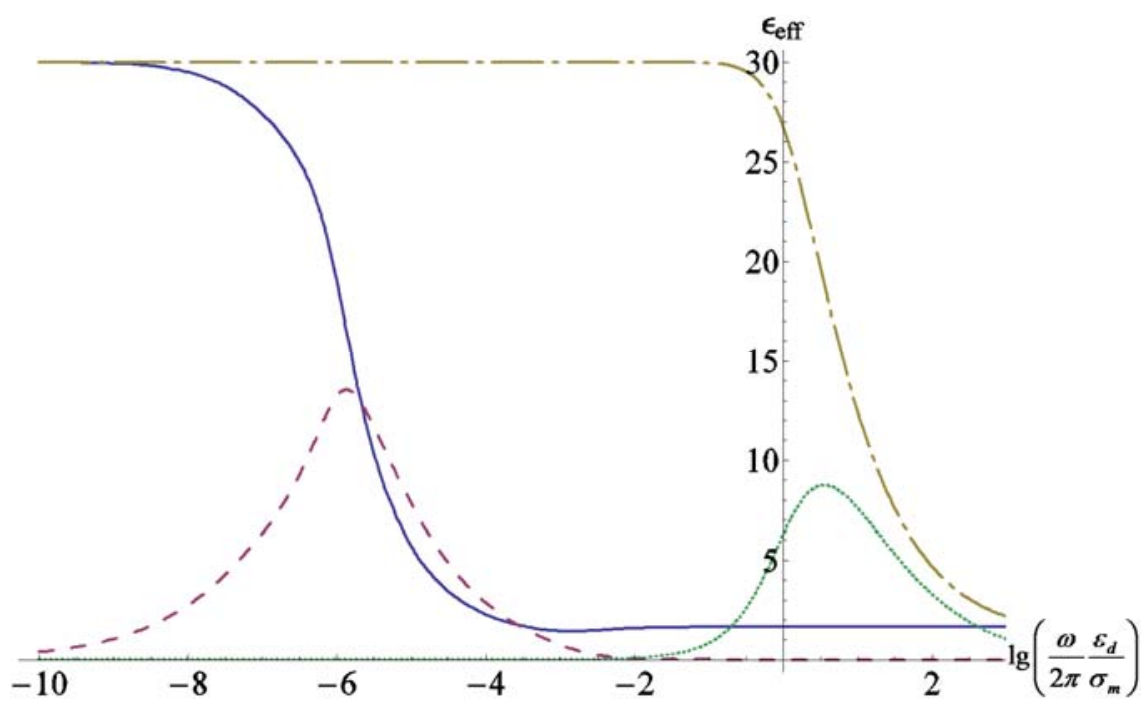

Fig. 1 The imaginary part of the effective permittivity versus the ratio of dielectric and metal conductivities at $\varepsilon_{0} \sigma_{m}=3 \cdot 10^{17} \mathrm{~Hz}, \varepsilon_{d}=3$. The dash-dotted curve presents a real part of permittivity calculated by EMT, the dotted curve presents an imaginary part of permittivity in the same case, the solid line presents the real part of permittivity calculated by MEMT, dashed curve presents an imaginary part of permittivity in the same case. The volume concentration of the inclusion is 0.3 .

It is obvious from Fig. 1 that it is indeed the inclusion's conductivity decrease caused by the skin effect that leads to the experimentally observed shift of the peak of losses towards the micro wave range [7, 10]. However, taking into account the skin-effect cannot explain the observed broadening of the maximum of losses.

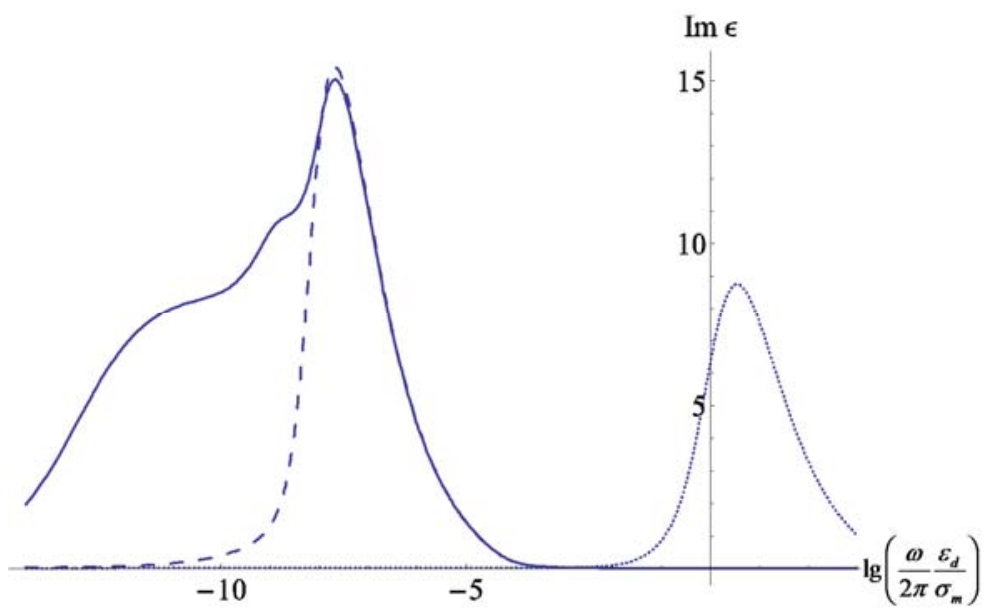

Fig. 2: The frequency dependence of the imaginary part of permittivity of the system without fluctuations calculated by EMT (dotted curve), MEMT (dashed curve) and of the imaginary part of permittivity of the system with fluctuations of concentration calculated by two step MEMT (solid curve).

To understand the nature of this broadening we should consider the role of concentration fluctuations in the composite medium. It is obvious that these fluctuations can significantly change the effective parameters of the system under consideration. It is commonly known that in order to 
obtain a material with a high permittivity value one has to consider composites in which the metal concentration is slightly below the percolation threshold. On the other hand, the closer the system to the percolation threshold the more significant is the role played by the fluctuations [1]. In particular, it may happen that in a certain region of the volume the inclusions' concentration $p_{l o c}$ slightly exceeds the percolation threshold. As a consequence, the eddy currents induced in that region will cause additional losses. Note that, due to technology imperfections, these fluctuations in inclusions' concentration are inevitable and may significantly exceed the fluctuations corresponding to uncorrelated random distribution. The origin of such fluctuations may be an insufficient mixing of the ingredients, attraction or repulsion between inclusions due to surface tension, etc.

To illustrate the speculations above we consider a simple model problem. For instance, let us compare the losses in two different mixtures of conducting particles embedded in a lossless dielectric matrix. The mean volume concentration $p_{0}$ of the conducting inclusions is the same in both systems. We assume $p_{0}$ to be less than the percolation threshold $p_{c}: p_{0}<p_{c}$. In the first system, the conducting inclusions are randomly distributed. In the second system we assume a correlated distribution of the conducting inclusions. Namely, we have randomly chosen regions (regions with high concentration of inclusions) of radius $a_{h c}$ where the concentration $p_{1}$ of the conducting inclusions exceeds the percolation threshold $p_{c}$ and, as a consequence, it also exceeds the mean value $p_{0}$ : i.e. $p_{1}>p_{c}>p_{0}$. We can consider the secondary percolation problem, which is the problem of percolation through the regions with high concentration of inclusions. The volume concentration $c_{l}$ of these regions is considered to be smaller than the corresponding percolation threshold $c_{c}$. The volume concentration $p_{2}$ outside these regions is thus slightly less than $p_{0}$ and $p_{c}$ :

$$
p_{2}=\left(p_{0}-c_{1} p_{1}\right) /\left(1-c_{1}\right)<p_{0}<p_{c} .
$$

To evaluate the values of the permittivity of the first system we employ the MEMT (Eq. (3)). For this purpose we have to define the radius $a_{d}$ of the "dielectric inclusions" imbedded in the matrix of the effective material. By employing the MEMT we assume that steady state approximation is still valid, namely, the wavelength in the matrix surrounding the inclusions is much greater than both the radius of the inclusions and the mean distance among them. As a consequence, the fields outside inclusions and inside dielectric inclusions are governed by the Laplace equation (this is not the case of metallic inclusions, inside which we have to solve the Maxwell equations). As a consequence, the radius of the regions filled with dielectric material drops out from the solution $\left(F\left(k_{d} a_{d}\right)<<1\right)$. For the sake of simplicity we put $a_{d}=a_{m}$.

For the second system we first evaluate the permittivity inside and outside the regions with increased concentrations employing MEMT and obtaining $\varepsilon_{e f f}\left(p_{1}\right)$ and $\varepsilon_{e f f}\left(p_{2}\right)$. For the second step of the calculation, we consider the volumes with high concentration $p_{1}$ as "conducting" inclusions, whose permittivity is equal to $\varepsilon_{\text {eff }}\left(p_{1}\right)$ and volume concentration is equal to $c_{1}$. The volume with low concentration is represented by equivalent "dielectric" inclusions, whose permittivity is equal to $\varepsilon_{\text {eff }}\left(p_{2}\right)$ and the volume concentration is equal to $c_{2}=1-c_{1}$. The inequality $p_{1}>p_{c}>p_{0}>p_{2}$ yields $\operatorname{Im} \varepsilon_{e f f}\left(p_{1}\right)>>\operatorname{Im} \varepsilon_{\text {eff }}\left(p_{0}\right)>\operatorname{Im} \varepsilon_{\text {eff }}\left(p_{2}\right)$. It means that the wavelength and skin depth in the low concentration (lc) medium are greater then those in the high concentration (hc) medium and we can steel use the Laplace equation to solve the problem for lc-regions and $a_{l c}$ drops out from the solution. Thus, we can put $a_{l c}=a_{h c}$ without loss of generality. Ultimately, we evaluate the effective permittivity of the mixture of such new inclusions employing MEMT.

The results of this evaluation are shown in Fig. 2. We have chosen the following parameters of fluctuations: $\left.p_{0}=0.3<p_{c}=1 / 3\right), p_{1}=0.335>p_{c}, c_{1}=0.2, a_{h c}=10 a_{d}=3 \cdot 10^{-5} \mathrm{~m}$. We 
can see that at some frequencies additional fluctuations may result in the increase of losses more than ten times. Thus, in order to achieve high values of permittivity with low losses one should get as uniform distribution of inclusions as possible in order to avoid the local concentration exceeding the percolation threshold.

It is worth noting that, in reality, this distribution can hardly be controlled. One may propose to study the surface of a sample. However, such a surface is a two-dimensional system and the percolation threshold of $2 \mathrm{D}$ systems is much greater than the percolation threshold of $3 \mathrm{D}$ systems. In other words, percolation channels will be observed neither within the high concentration medium, nor in the low concentration medium. Thus, both regions will look like being below the percolation threshold.

\section{Conclusion}

It is shown that if the effective skin depth in the regions occupied by the fluctuation is comparable to the mean diameter of these regions we can expect additional losses. Thus, the fluctuations in conducting inclusion concentration may be responsible for the well-known disagreement between the theory and experiment in microwave range: the theories (percolation theory, effective medium theory etc.) predict much lower losses than those measured in experiment [10]. To evaluate the losses we propose a two-step procedure that takes into account the skin depth effect both on separate inclusion and on density.

\section{Acknowledgments}

The work was performed with the support of RFBR projects No. 08-02-00874, and No. 07-02-91583. We also thank A. V. Dorofeenko for his kind collaboration.

\section{References}

[1] B. I. Shklovskii, A. L. Efros, Electronic Properties of Doped Semiconductors (Springer, NY, 1989).

[2] A. P. Vinogradov, A. M. Karimov, A. T. Kunavin, A. N. Lagarkiv and A. K. Sarychev, Sov. Phys. Dokl DAN SSSR, 275, 590 (1984).

[3] A. P. Vinogradov, Electrodynamics of composite materials (Editorial URSS, Moscow, 2001) (in Russian).

[4] L. D. Landau, E. M. Lifshitz and L. P. Pitaevskii, Electrodynamics of Continuous Media, 2nd ed., (Butterworth Heinemann, Oxford 1995)

[5] A.P. Vinogradov, A.V. Aivaziyan, Phys. Rev. E 60, 987-993 (1999)

[6] L. Lewin Theory of waveguides (Newnes-Butterworths, London, 1975).

[7] A. P. Vinogradov, L. P. Panina, A. K. Sarychev, Sov. Phys. Dokl. 36, 530- 532 (1989).

[8] I. Vendik, O. Vendik, M. Gashinova, I. Kolmakov, M. Odit, L. Jylhä, S. Maslovski, S. Tretyakov, O. Ouchetto, and S. Zouhdi, in Proc. of Progress in Electromagnetics Research Symposium, Cambridge, USA, 26-29 March 2006, p.8

[9] A. P. Vinogradov, A. V. Dorofeenko, S. Zouhdi, Physics - Uspekhi 51 (5) 485 - 492 (2008)]

[10] K. N. Rosanov, PhD thesis, Moscow IVT AN (1991). 